\title{
The symbiotic star H1-36
}

\section{A composite model of line and continuum spectra from radio to ultraviolet}

\author{
R. Angeloni ${ }^{1,2}$, M. Contini ${ }^{2,1}$, S. Ciroi ${ }^{1}$, and P. Rafanelli ${ }^{1}$ \\ 1 Dipartimento di Astronomia, Università di Padova, Vicolo dell'Osservatorio 2, 35122 Padova, Italy \\ e-mail: [rodolfo.angeloni ; stefano.ciroi;piero.rafanelli]@unipd.it \\ 2 School of Physics and Astronomy, Raymond and Beverly Sackler Faculty of Exact Sciences, Tel Aviv University, \\ Tel Aviv 69978, Israel \\ e-mail: contini@post.tau.ac.il
}

Received 22 March 2007 / Accepted 2 May 2007

\section{ABSTRACT}

\begin{abstract}
Aims. In this paper, we analyse the spectra of D-type SS H1-36 within a colliding-wind scenario. We aim to analyse the properties of this object by taking the observational data along the whole electromagnetic spectrum into account, in order to derive a self-consistent picture able to interpret the nature of the system as a whole.

Methods. After constraining the relative physical conditions by modelling more than 40 emission lines from radio to UV, we are able to explain the continuum spectral energy distribution by considering all the emitting contributions arising from both the stars, the dust shells, and the gaseous nebulæ.

Results. A comprehensive model of the radio spectra allows reproduction of the different slopes of the radio profile and the turnover frequency, as well as the different sizes of the observed shocked envelope at different frequencies in the light of the contributions from the expanding and reverse nebulæ. The IR continuum unveils the presence of two dust shells with different radii and temperatures, which might be a distinctive feature of D-type symbiotic systems as a class of objects. The broad profiles of IR lines direct us to investigate whether an X-ray jet may be present.

Conclusions. This insight leads us to indicate H1-36 as a promising X-ray target and to encourage observations and studies that consistently take the complex nature of symbiotic stars into account throughout the whole electromagnetic spectrum.
\end{abstract}

Key words. binaries: symbiotic - stars: individual: H1-36

\section{Introduction}

Symbiotic systems (SS) are currently understood as interacting binaries composed of a compact star, generally but not necessarily a white dwarf (WD) that is the source of the ionizing radiation, a cool giant star, which is at the origin of dust formation and ejection episodes, and different emitting gas and dust nebulæ. The wide variety of the properties of these systems often makes their classification as a single group difficult; however, these differences are probably more related to distinct physical conditions like the masses, orbital periods, and temperature of the hot source, than different kinds of phenomena.

Because of the associated circumstellar matter and energetic activity, many SS emit detectable radiation nearly across the entire electromagnetic spectrum. On the basis of near-infrared (NIR) colours, SS were classified in S and D types (Webster \& Allen 1975) according to whether the cool star (S-type) or dust (D-type) dominates the $1-4 \mu \mathrm{m}$ spectral range. The continuum radio emission has been modelled as thermal bremsstrahlung radiation arising from (a) the photoionized component of the cool star wind (Seaquist et al. 1984, hereafter STB; Taylor \& Seaquist 1984); (b) a wind associated with a slow nova-like eruption from the hot companion; (c) the interaction region involving both winds in mutual collisions (Watson et al. 2000; Kenny \& Taylor 2005; Bisikalo et al. 2006). Whatever the case, it is widely recognised that the radio spectra provide unique information about the mass-loss phenomena at the base of symbiotic activity. Eventually, there is growing evidence that SS can be the site for jet structures and synchrotron emission, confirming the primary role of shocks in the now widely accepted interpretation of SS as colliding-wind binary systems.

H1-36 was among the most interesting objects in the extensive Purton radio survey of emission-line stars (1982). The particular attention that was drawn to it since Haro's discovery (1952) was due to a curious set of misunderstood and genuine insights: catalogued as planetary nebula despite its extremely high-excitation emission-line spectrum and its imposing infrared excess, H1-36 was even thought to be the optical counterpart of the Uhuru hard X-ray source 3U 1746-37 (Giacconi et al. 1974). Finally, Allen permanently classified H1-36 as a D-type SS by including it in his catalogue (Allen 1981) and dedicating a paper to it (Allen 1983) that so far still represents the most complete observational work on this object.

One of the most important results emerging from that study is that the Mira star experiences an extraordinary reddening $\left(A_{\mathrm{v}} \sim 20 \mathrm{mag}\right)$, especially when compared to the one towards the emission-line regions (only $2.2 \mathrm{mag}$ ). This allowed Allen to point out that the cool component is heavily embedded in its own circumstellar dust shell, illuminated from the outside by a $T_{*} \sim 150000 \mathrm{~K}$ star. The derived cool star and dust temperatures would then be, respectively, $T_{\mathrm{M}} \sim 2500 \mathrm{~K}$ and $T_{\mathrm{d}} \sim 700-800 \mathrm{~K}$.

Another interesting feature of $\mathrm{H} 1-36$ lies in the radio range. As a matter of fact, it is one of a small number of radio sources whose spectra are flat at high frequencies but that turn towards 
Table 1. The main parameters of the H1-36 D-type symbiotic star.

\begin{tabular}{ccc}
\hline \hline- & - & Ref. \\
\hline$\alpha(\mathrm{J} 2000)$ & 174948.1 & Belczyński et al. (2000) \\
$\delta(\mathrm{J} 2000)$ & -370127.9 & Belczyński et al. (2000) \\
WD temperature & $1.5 \times 10^{5} \mathrm{~K}$ & Allen (1983) \\
Cool star spectrum & Mira $-\mathrm{M} 4-\mathrm{M} 5$ & Allen 83 - Medina-Tanco \& Steiner (1995) \\
Mira temperature & $2500 \mathrm{~K}$ & Allen (1983) \\
Mira pulsation period & $450-500 \mathrm{~d}$ & Whitelock (1987) \\
Dust temperature & $700 \mathrm{~K}$ & Allen (1983) \\
Distance & $4.5 \mathrm{kpc}$ & Allen (1983) \\
Binary separation & $3 \times 10^{16} \mathrm{~cm}$ & Allen (1983) \\
Orbital period & $\geq 10 \mathrm{D}^{3 / 2} \mathrm{yr}$ & Allen (1983) \\
Other names & PK 353-04 1=Hen2-289= & Belczyński et al. (2000) \\
& $=$ Haro 2-36=IRAS 17463-3700 & \\
\hline
\end{tabular}

a spectral index near +1 at lower frequencies (Purton 1977). Several papers hence tried to explain this behaviour in terms of free-free emission from the ionized cool star wind, unfortunately without considering the possibility of any outflow from the compact star (for a further description of this models, see Sect. 4.2.1).

The papers that present both imaging (e.g. Bhatt \& Sagar 1991; Corradi et al. 1999, who resolved the complex nebula at radio and optical wavelengths) and new spectra (e.g. Costa \& de Freitas Pacheco 1994; Pereira 1995; Pereira et al. 1998) have not substantially modified the original scenario drawn by Allen's analysis, whose physical parameters still have to be considered as the most reliable ones available in the literature. Interestingly, H1-36 is the only SS known to support OH- (Ivison et al. 1995), as well as $\mathrm{SiO}-$ (Allen 1989) and $\mathrm{H}_{2} \mathrm{O}$-masers (Ivison et al. 1998).

In this paper we aim to model H1-36 (Table 1) by combining literature observations from radio to UV collected over a period of about 30 years (Sect. 2). Within a colliding-wind scenario and by means of the SUMA code (Sect. 3), we start by modelling more than 40 emission lines from IR to UV in order to constrain the physical conditions across the whole system (shocked nebulæ, dust shells, hot and Mira stars). In the light of the derived parameters, we are then able to fit the composite continuum SED in a self-consistent way. The results of this cross-checked method are extensively presented in Sect. 4. Concluding remarks appear in Sect. 5.

\section{Observational details}

\subsection{Radio-mm observations}

In Table 2 we present the data we collected from the literature in order to ensure as much a complete spectral coverage in the radio-mm range as possible. The most important references to the H1-36 radio spectrum remain Purton's papers (Purton 1977, 1982), in which observations from about $2 \mathrm{GHz}$ to $90 \mathrm{GHz}$ obtained with the CSIRO 64-m telescope at Parkes, Australia, the NRAO 11-m telescope at Kitt Peak, USA, and the NRAO threeelement interferometer at Green Bank, USA, are presented. In the next years several works, focused mainly on the mass loss properties of the cool stellar component (e.g. Jones 1985) and on the presumed correlations among different spectral bands (Seaquist et al. 1993), performed observations at intermediate frequencies. They allowed us to extract a well-defined radio profile and eventually to confirm that the object has not been significantly variable in the last 30 years.
Table 2. The radio-mm data references.

\begin{tabular}{ccc}
\hline \hline$v[\mathrm{GHz}]$ & Flux [mJy] & Ref. \\
\hline 0.843 & $10.6 \pm 1.2$ & Jones (1985) \\
1.4 & 13.5 & Siodmiak \& Tylenda (2001) \\
1.6 & 15 & Taylor \& Seaquist (1984) \\
2.7 & $33 \pm 4$ & Purton et al. (1982) \\
4.885 & 50 & Aaquist \& Kwok (1990) \\
5.0 & $46 \pm 10$ & Purton et al. (1982) \\
5.0 & 50 & Siodmiak \& Tylenda (2001) \\
6.2 & $65 \pm 10$ & Purton et al. (1982) \\
8.1 & $82 \pm 7$ & Purton et al. (1982) \\
8.31 & 73.5 & Bastian (1992) \\
8.44 & $65.3 \pm 3.3$ & Seaquist et al. (1993) \\
8.9 & $90 \pm 15$ & Purton et al. (1982) \\
14.5 & $90 \pm 10$ & Purton et al. (1982) \\
14.965 & 72 & Aaquist \& Kwok (1991) \\
90.0 & $127 \pm 53$ & Purton et al. (1982) \\
231 & $55 \pm 16$ & Ivison et al. (1995) \\
\hline
\end{tabular}

This ensures that collecting data coming from a wide temporal range is, at least for this SS, a reliable approach. Summarising, we are able to present a radio-mm profile composed of 16 points from $843 \mathrm{MHz}$ to $230 \mathrm{GHz}$ (Fig. 1, bottom panel).

\subsection{Infrared data}

D-type SS are known to be variable systems in the IR range, where we observe the contributions of different emitting components, i.e. cool star and dust shells. Quite surprisingly, as in the radio case, the H1-36 IR spectra do not show dramatic variations in the data flux over the years we are going to discuss (Table 3 , Fig. 1, top panel). Indeed, IRAS points (1982) agree well with ISO-SWS spectrum (taken in 1996) and with the $34.6 \mu \mathrm{m}$ measurement presented in He et al. (2005), and the accordance with the MSX6C photometric points (Egan et al. 2003) is also reasonably good. At NIR wavelengths, the Allen's NIR bands are slightly shifted with respect to the DENISE and 2MASS bands, especially the $H$ and $J$ bands, which feel the increasing contribution of the cool star. This is confidently due to the Mira variations, whose period is about 450-500 days (Whitelock 87).

\subsection{Optical spectra and UV spectrophotometry}

Also in this case the analysed data are a collection of different observing sessions from 1975 to 1979 . These are presented in Allen (1983), where the reader is sent for further technical 
Table 3. The infrared data references.

\begin{tabular}{ccc}
\hline \hline$\lambda[\mu \mathrm{m}]$ (phot. band) & ${\text { Flux [Jy }]^{a}}^{a}$ & Ref. \\
\hline $1.20(J), 1.64(H), 2.22(K), 3.8\left(L^{\prime}\right)$ & $1.55-2.1-4.8-7.1$ & Allen (1983) \\
$8.28,12.13,14.65$ & $7.454-11.93-14.67$ & MSX6C PSC, Egan et al $^{b}(2003)$ \\
$1.235\left(J^{*}\right), 1.662\left(H^{*}\right), 2.159\left(K^{*}\right)$ & $0.0312-0.115-0.594$ & Ramos-Larios \& Phillips $^{c}(2005)$ \\
$0.9\left(I_{\mathrm{c}}\right), 1.25(J), 2.22(K)$ & $0.0167-0.034-0.741$ & DENISE $^{d}$ \\
$12,25,60$ & $18.13-28.16-5.75$ & IRAS PSC $^{e}$ \\
34.6 & 16.6 & ${\text { He et al. }(2005)^{f}}^{f}$ \\
2.5-45.5 SWS & --- & ISO IDA $^{g}$ \\
\hline
\end{tabular}

* 2MASS Photometric System; ${ }^{a}$ out of Allen, expressed in $10^{14} \mathrm{erg} \mathrm{s}^{-1} \mathrm{~cm}^{-2} \mathrm{~A}^{-1}{ }^{b}$ the Midcourse Space Experiment Point Source Catalog Version 2.3 (October 2003); ${ }^{c}$ 2MASS NIR measurements of galactic planetary nebulae; ${ }^{d}$ the DENIS consortium (third release - Sept. 2005); ${ }^{e}$ IRAS Catalogue of Point Sources, Version 2.0 (IPAC 1986); ${ }^{f}$ the $35 \mu \mathrm{m}$ absorption line towards $1612 \mathrm{MHz}$ masers; ${ }^{g}$ ISO Data Archive (@ http://www.iso.vilspa.esa.es).

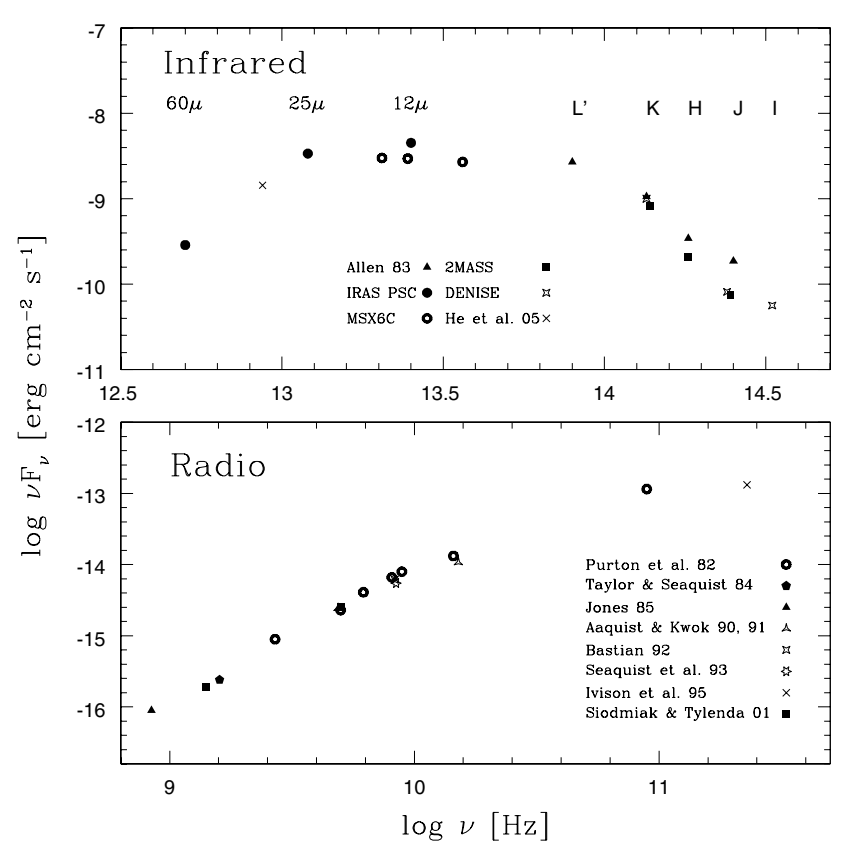

Fig. 1. Observational data. Top panel: IR spectral range - for sake of clarity we do not show the ISO spectrum. Bottom panel: radio spectral range.

details. Moreover, it is explicitly stated in that paper that the optical data "are combined in the belief that variability of the lines is slight". This has been reconfirmed by recent spectra, which in fact have not shown relevant changes in the line and continuum flux level (Costa \& de Freitas Pacheco 1994; Pereira 1995; Pereira et al. 1998).

\section{The theoretical framework}

\subsection{The colliding-wind scenario}

In the past years, theoretical models (Girard \& Willson 1987; Kenny \& Taylor 2005), as well as observations (Nussbaumer et al. 1995), have categorically shown that in SS both the hot and cool stars lose mass through strong stellar winds that collide within and outside the system, thereby creating a complex network of wakes and shock fronts that result in a complicated structure of gas and dust nebulæ (Nussbaumer 2000).

In this paper, as previously done for other SS (see Sect. 3.2), we refer to two main shocks: the shock between the stars facing the WD, which is a head-on shock (hereafter the reverse shock); and the head-on-back shock, which propagates outwards the system (hereafter the expanding shock). Both the nebulae downstream of the shock fronts are ionized and heated by the radiation flux from the hot star and by shocks. The photoionizing radiation flux reaches the very shock front of the reverse shock, while it reaches the edge opposite to the shock front downstream of the expanding shock.

\subsection{The SUMA code}

The results presented in this work were performed with SUMA (Viegas \& Contini 1994; Contini 1997), a numerical code that simulates the physical conditions of an emitting gaseous cloud under the coupled effect of ionization from an external radiation source and shocks and in which both line and continuum emission from gas are calculated consistently with dustreprocessed radiation (grain heating and sputtering processes are also included). The derived models have been successfully applied to several SS: e.g. AG Peg (Contini 1997, 2003), HM Sge (Formiggini et al. 1995), RR Tel (Contini \& Formiggini 1999), He2-104 (Contini \& Formiggini 2001), R Aqr (Contini \& Formiggini 2003), HD330036 (Angeloni et al. 2007b), as well as to nova stars (V1974 Cyg, Contini et al. 1997 - T Pyx, Contini \& Prialnik 1997) and supernova remnants (Kepler's SNR, Contini 2004).

The calculations start with gas and dust entering the shock front in a steady state regime: the gas is adiabatically compressed and thermalized throughout the shock front. In the downstream region the compression is derived by solving the RankineHugoniot equations (Cox 1972): the downstream region is automatically divided in plane parallel slabs in order to calculate the physical conditions throughout the nebula as smoothly as possible. Radiation transfer and optical depths of both continuum and lines are calculated for a steady state: in particular, radiation transfer of the diffused radiation is taken into account following Williams (1967). The fractional abundance of the ions in different ionization stages is calculated in each slab by solving the ionization equilibrium equations for the elements $\mathrm{H}, \mathrm{He}, \mathrm{C}, \mathrm{N}$, $\mathrm{O}, \mathrm{Ne}, \mathrm{Mg}, \mathrm{Si}, \mathrm{S}, \mathrm{Cl}, \mathrm{Ar}$, and Fe. The electron temperature in each slab is obtained from the energy equation when collisional processes prevail and by thermal balancing when radiation processes dominate.

Compression downstream strongly affects the gas cooling rate by free-free, free-bound, and line emission; consequently, the emitting gas will have different physical conditions depending on the shock velocity and on the pre-shock density. For example, self-absorption of free-free radiation, consistently calculated in every slab, affects the emission spectral index at low radio frequencies (see Sect. 4.2.1) 
Dust is included in the calculations, too. Dust and gas are coupled throughout the shock-front and downstream by the magnetic field. In each slab the sputtering of the grains is calculated, leading to grain sizes that depend on the shock velocity and on the gas density. The temperature of the grains, which depends on the grain radius, is calculated by radiation heating from the external (primary) source and by diffuse (secondary) radiation, as well as by gas collisional heating. The dust-reprocessed radiation flux is calculated by the Plank-averaged absorption coefficient of dust in each slab and integrated throughout the nebula downstream.

The input parameters that characterise the shock are the shock velocity, $V_{\mathrm{s}}$, the preshock density of the gas, $n_{0}$, and the preshock magnetic field, $B_{0}$. The radiation flux is determined by the temperature of the star, interpreted as a colour temperature, $T_{*}$, and by the ionization parameter, $U$. The dust-to-gas ratio, $d / g$ is also accounted for, as well as the relative abundances of the elements to $\mathrm{H}$.

A full detailed description of the code is to be presented in Contini \& Viegas (2007, in preparation).

\section{Modelling the spectra}

In this section we present those models that best reproduce the whole properties of H1-36. The models dealing with the observed line spectra appear in Sect. 4.1. They are cross-checked by the continuum SED (Sect. 4.2) until a fine tuning of line and continuum spectra is found. We refer in particular to the SED in the radio range (Sect. 4.2.1), where the turnover frequency and the steeper spectral index of the bremsstrahlung at lower frequencies are highly sensitive to the physical conditions of the gas.

\subsection{The line ratios}

The spectra derived from Allen's observations are shown in Table 4, Col. 2; the models calculated for the reverse $\left(m_{\mathrm{rev}}\right)$ and for the expanding shock $\left(m_{\exp }\right)$ are presented in Cols. 3 and 4 , respectively, and their weighted sum appears in Col. 5.

The input parameters of the models appear in Table 5. They are constrained by the observations, namely, the shock velocity $V_{\mathrm{s}}$ is roughly determined by the FWHM of the line profiles (in agreement with Allen 1983); the preshock density $n_{0}$ is constrained by the slope of the radio continuum and by the ratios of characteristic lines (e.g. [SII] 6717/6731); the preshock magnetic field $B_{0}$ is characteristic of symbiotic systems (Crocker et al. 2001); the stellar temperature $T_{*}$ is taken from the analysis of Allen (1983); the ionization parameter $U$ is determined phenomenologically; the geometrical thickness $D$ is constrained by the radio continuum slope and by the ratios of the lines to $\mathrm{H} \beta$; eventually, the relative abundances of the elements are determined by the line ratios. Unfortunately, in the present case, the dust-to-gas ratio $(d / g)$ cannot be directly determined because the bump of the dust reprocessed radiation is covered by the emission from the summed dust shell and Mira black body fluxes.

The line ratios calculated by $m_{\mathrm{rev}}$ and $m_{\mathrm{exp}}$ are summed up after adopting the weights $w$ shown in the last row of Table 5 . They are the same as those adopted in Fig. 3 to suite the continuum SED.

The observed line ratios are reproduced by the summed model within a factor of 2 , except a few lines that are badly fitted, e.g. [NI] 5200, since they have a particularly low critical density for collisional deexcitation and are very faint at the
Table 4. The fluxes of the UV and optical spectral lines, normalized to $\mathrm{H} \beta$.

\begin{tabular}{ccccc}
\hline \hline Line & Obs $^{a}$ & $m_{\text {rev }}$ & $m_{\exp }$ & $a v_{m_{\text {rev }}+m_{\text {exp }}}$ \\
\hline CIV 1549 & 18.78 & 19.7 & 27.3 & 22 \\
HeII 1640 & $7.88^{b}$ & 1.1 & 5.2 & 2.6 \\
NIII] 1749 & 5.15 & 2.24 & 2. & 2.2 \\
CIII] 1909 & 27.2 & 9.3 & 18. & 13.0 \\
[NeV]+OIII 3425 & 4.24 & $0.13^{c}$ & $0.13^{c}$ & $0.22^{c}$ \\
[FeVI] 3662 & 0.006 & 0.0043 & 0.0047 & 0.0044 \\
[OII]+ 3727 & 0.576 & 0.04 & 2.6 & 0.99 \\
[NeIII] 3869+ & 2.12 & 1.5 & 2.2 & 1.78 \\
[SII]+ 4070 & 0.18 & 0.38 & 0.08 & 0.27 \\
H $\gamma$ & 0.42 & 0.44 & 0.45 & 0.44 \\
[OIII] 4363 & 0.61 & 1.29 & 0.80 & 1.1 \\
HeI 4471 & 0.056 & 0.036 & 0.023 & 0.044 \\
[FeIII] 4658 & 0.015 & 0.01 & 0.015 & 0.012 \\
HeII 4686 & 0.55 & 0.16 & 0.76 & 0.4 \\
H $\beta$ 4861 & 1 & 1 & 1 & 1 \\
[ArIV] 4711 & 0.02 & 0.0013 & 0.13 & 0.049 \\
[NeIV]+ 4714 & 0.11 & 0.14 & 0.08 & 0.12 \\
[ArIV] 4740 & 0.097 & 0.011 & 0.15 & 0.063 \\
[OIII 5007+ & 19.1 & 2. & 35. & 14.2 \\
[FeVI] 5146 & 0.017 & 0.007 & 0.03 & 0.016 \\
[FeII] 5159 & 0.03 & 0.039 & 0.0017 & 0.025 \\
[FeVI] 5176 & 0.03 & 0.018 & 0.033 & 0.023 \\
[ArIII]5191 & 0.005 & 0.017 & 0.012 & 0.015 \\
[NI] 5199 & 0.006 & 0.0002 & 0.0005 & 0.0003 \\
[FeVI] 5335 & 0.011 & 0.0018 & 0.017 & 0.008 \\
[NII] 5755 & 0.15 & 0.43 & 0.04 & 0.28 \\
HeI 5876 & 0.11 & 0.18 & 0.07 & 0.14 \\
[FeVII] 6087 & 0.12 & 0.002 & 0.005 & 0.003 \\
[OI] 6300+ & 0.4 & 0.47 & 0.013 & 0.3 \\
[SIII] 6312 & 0.08 & 0.14 & 0.13 & 0.136 \\
[NII] 6548+ & 1.0 & 0.2 & 1.4 & 0.63 \\
H $\alpha$ & 4.5 & 3.5 & 3.1 & 3.4 \\
[SII] 6717 & 0.02 & 0.002 & 0.057 & 0.022 \\
[SII] 6731 & 0.039 & 0.0035 & 0.10 & 0.039 \\
[ArIII] 7136 & 0.24 & 0.14 & 0.24 & 0.14 \\
[ArIV] 7170 & 0.007 & 0.023 & 0.009 & 0.018 \\
[OII] 7319+ & 0.55 & 2.6 & 0.48 & 1.8 \\
[FeII] 8617 & 0.0027 & 0.0027 & 0.0011 & 0.002 \\
[SIII] 9069 & 0.33 & 0.06 & 1.3 & 0.5 \\
H $\beta$ abs. ${ }^{d}$ & - & 1.13 & 0.0075 & - \\
\hline
\end{tabular}

${ }^{a}$ Observed values from Allen (1983); ${ }^{b}$ blended with OIII 1640; ${ }^{c}$ the calculated flux refers only to [NeV]; ${ }^{d} \mathrm{H} \beta$ absolute flux calculated at the nebulæ $\left[\mathrm{erg} \mathrm{cm}^{-2} \mathrm{~s}^{-1}\right]$.

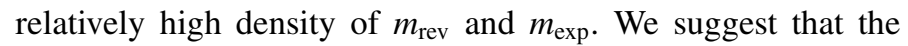
observed HeII 1640/ $\mathrm{H} \beta$ may be blended with the rather high OIII $1640 / \mathrm{H} \beta$ ratio, whose calculated value is $>3$. An improved fit of the [OIII] 5007/[OIII] 4363 ratio could result from a reverse shock with lower densities, which are however incompatible with the radio SED. As a matter of fact, the [OII] 3727 and [OIII] 5007 lines are mostly emitted from the expanding shock.

Model $m_{\text {rev }}$ shows $\mathrm{N} / \mathrm{H}$ and $\mathrm{O} / \mathrm{H}$ slightly higher than solar by factors of 1.7 and 1.3 , respectively, while Fe is strongly depleted both in $m_{\text {rev }}$ by a factor of 10 and in $m_{\exp }$ by a factor of 5 . This is not surprising as iron, a refractory element, is easily trapped into dust grains.

In Fig. 2 we present the profile of the electron density, electron temperature, and the fractional abundances of the most significant ions throughout the nebula downstream of the reverse (left diagram) and expanding shock (right diagram). It is worth noticing that model $m_{\mathrm{rev}}$ is matter-bound. This is characteristic of 

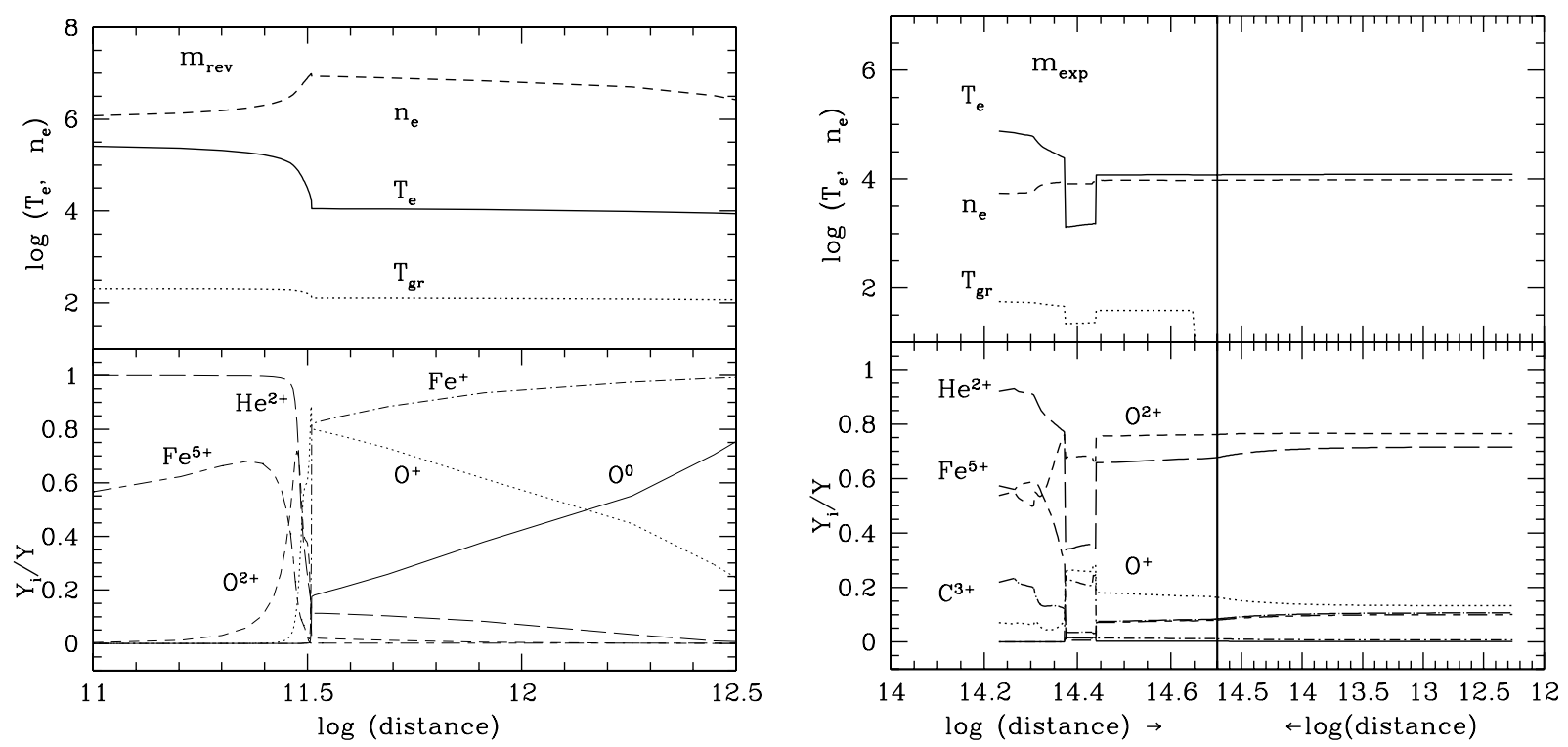

Fig. 2. The profile of the electron temperature, of the electron density, and of the temperature of the grains $\left(a_{\mathrm{gr}}=0.2 \mu \mathrm{m}\right)$ downstream of the reverse (left diagram) and of the expanding shock front (right diagram). The stratification of the most significant ions is shown in the bottom panels. For both diagrams, the shock front is on the left side.

Table 5. The model input parameters.

\begin{tabular}{ccc}
\hline \hline Parameter & $m_{\text {rev }}$ & $m_{\text {exp }}$ \\
\hline$V_{\mathrm{s}}\left(\mathrm{km} \mathrm{s}^{-1}\right)$ & 140 & 70 \\
$n_{0}\left(\mathrm{~cm}^{-3}\right)$ & $2.5 \mathrm{e} 5$ & $3.5 \mathrm{e} 3$ \\
$B_{0}(\mathrm{Gauss})$ & $1 \mathrm{e}-3$ & $1 \mathrm{e}-3$ \\
$T_{*}(\mathrm{~K})$ & $1.5 \mathrm{e} 5$ & $1.5 \mathrm{e} 5$ \\
$U$ & $2 \mathrm{e}-3$ & $2.5 \mathrm{e}-3$ \\
$D(\mathrm{~cm})$ & $2.8 \mathrm{e} 12$ & $1 \mathrm{e} 15$ \\
$d / g$ & $1 \mathrm{e}-14$ & $1 \mathrm{e}-14$ \\
$\mathrm{He} / \mathrm{H}$ & 0.1 & 0.1 \\
$\mathrm{C} / \mathrm{H}$ & $3.3 \mathrm{e}-4$ & $3.3 \mathrm{e}-4$ \\
$\mathrm{~N} / \mathrm{H}$ & $1.5 \mathrm{e}-4$ & $9.1 \mathrm{e}-5$ \\
$\mathrm{O} / \mathrm{H}$ & $8.6 \mathrm{e}-4$ & $6.6 \mathrm{e}-4$ \\
$\mathrm{Ne} / \mathrm{H}$ & $8.3 \mathrm{e}-5$ & $8.3 \mathrm{e}-5$ \\
$\mathrm{Mg} / \mathrm{H}$ & $2.6 \mathrm{e}-5$ & $2.6 \mathrm{e}-5$ \\
$\mathrm{Si} / \mathrm{H}$ & $3.3 \mathrm{e}-5$ & $3.3 \mathrm{e}-5$ \\
$\mathrm{~S} / \mathrm{H}$ & $1.6 \mathrm{e}-5$ & $1.6 \mathrm{e}-5$ \\
$\mathrm{Ar} / \mathrm{H}$ & $6.3 \mathrm{e}-6$ & $6.3 \mathrm{e}-6$ \\
$\mathrm{Fe} / \mathrm{H}$ & $3.2 \mathrm{e}-6$ & $6.2 \mathrm{e}-6$ \\
$\log w$ & -11.05 & -9.1 \\
\hline
\end{tabular}

the interbinary symbiotic nebulae, where the geometrical thickness is constricted by the colliding wind region.

\subsection{The continuum SED}

In Fig. 3 we present the modelling of the continuum. The SED is the result of the emitting contributions from the cool and hot stars, as well as of the fluxes from the dust shells and of the bremsstrahlung from the shocked nebulae downstream of the shock fronts, which emit the UV and optical line spectra.

The bremsstrahlung from the nebulæ shows two main peaks. At higher frequencies, the continuum is emitted from gas collisionally heated by the shock at relatively high temperatures; therefore, the peak frequency depends on the shock velocity. The peak at $\sim 10^{14} \mathrm{~Hz}$, on the other hand, depends on the volume of gas at temperatures of $\sim 1-3 \times 10^{4} \mathrm{~K}$, which is heated and ionized mainly by the photoionizing flux; therefore, the peak frequency is more sensitive to the radiation parameters $T_{*}$ and $U$. Also a contribution to the SED by synchrotron radiation, not so exotic in objects where shocks are at work, cannot be ruled out (see Sect. 4.2.1 and Fig. 3).

Without more constraining data, it remains puzzling to determine whether the measured flux densities at $\sim 10^{11} \mathrm{~Hz}$ are dominated by synchrotron, by bremsstrahlung, or by thermal emission from cold dust, also taking into account that emission from either mechanism could fluctuate in response to variable mass-loss episodes.

\subsubsection{The radio continuum}

In an important paper on radio emission of mass-losing stars, Wright \& Barlow (1975) presented an analytic study of the spectral flux distribution produced by completely ionized, uniform, spherically symmetric mass-loss flow in early type stars. Alhough using an idealised hypothesis, they tried to interpret the radio data for some recently observed objects in light of thermal free-free emission, which would result in a power-law spectrum $S_{v} \propto v^{0.6}$. They also discussed deviations from the ideal treatment caused by e.g., non-uniform mass-loss rates or by the actual ionization structure of the emitting circumstellar envelope.

In effect, the Purton's radio survey (Purton 1982) pointed out that there were some objects (with a high incidence of SS) whose radio spectral index was systematically steeper than $\alpha=0.6$ (in the range $0.8-1.5$ ), therefore implying an envelope density profile quite far from the inverse-square one assumed by Wright \& Barlow (1975). In any event, no attempt was made by Purton to fit a detailed physical model to the data.

A few years later, Seaquist et al. (1984) and Taylor \& Seaquist (1984) presented a model (hereafter STB model) that explained the H1-36 radio properties in light of the mounting evidence of the binary nature of SS: by assuming that the emitting region arises from the portion of the cool component wind photoionized by the WD, they performed a fit to the Purton's data with a least-square criterion as a function of the so-called $\mathrm{X}$ parameter (related to the shape of the ionized nebula), of the turnover frequency $v_{\mathrm{t}}$, and of the flux density at $v_{\mathrm{t}}$. The model was successful in reproducing many of the observed 


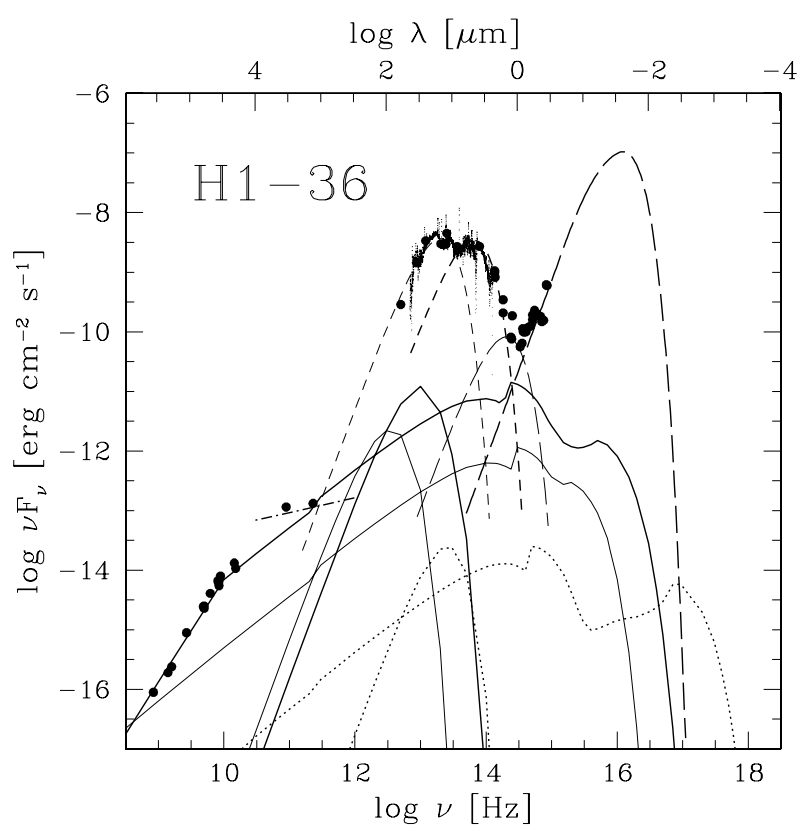

Fig. 3. The H1-36 continuum SED, from radio to UV. Thin shortdashed line: $250 \mathrm{~K}$ dust shell; thick short-dashed line: $800 \mathrm{~K}$ dust shell; thin long-dashed line: Mira $(2500 \mathrm{~K})$ stellar component; thick longdashed line: hot stellar component; dot-dashed line: synchrotron; thin solid line: $m_{\text {exp }}$; thick solid line: $m_{\text {rev }}$ (see Table 5 for details about the model input parameters); dotted line: the jet (see Sect. 4.3). Notice that for $m_{\mathrm{rev}}, m_{\mathrm{exp}}$, and the jet models, there are two curves: one the bremsstrahlung emission from radio to X-rays, the other the consistently calculated reprocessed radiation by dust in the IR.

properties of radio symbiotics, such as the spectral index, the spectral turnover, the correlation between the radio emission and the spectral type of the cool component. Nonetheless, it was soon recognised that the actual radio sources were more complex than those assumed in the STB model and that any derived physical property had to be treated with particular caution. Furthermore, more recent observations by Seaquist \& Taylor (1993) at millimeter and submillimeter wavelengths showed that several objects might differ considerably from the STB model.

As for the specific case of H1-36, the STB model failed e.g. to reproduce the $843 \mathrm{MHz}$ flux presented by Jones (1985). Moreover, the assumed density profile appeared too simplistic: even the two-layer model discussed by Costa \& de Freitas Pacheco (1994), referring to the inner structure of the emitting nebulae, cannot be considered adequate since SS have such large density gradients that do not even allow unique values to be adopted for electron temperature and densities.

Unfortunately, so far there have been no papers that try to interpret the radio spectra by considering the possibility of any outflow from the compact star.

In the following we fit the existing radio-mm data we have presented in Table 3 and in the bottom panel of Fig. 1, within the colliding-wind scenario depicted in Sect. 3.1. Our approach is shown to be self-consistent with the continuum profile of the system along the whole electromagnetic range, as well as being cross-checked by the spectral line emission.

Three main slopes are seen between $10^{8}$ and $10^{11} \mathrm{~Hz}$. The flattest one, with a spectral index of 0.75 , can be explained by the synchrotron radiation created by the Fermi mechanism at the shock front (Bell 1978a,b). A cut-off at the lower limit is given by Ginzburg \& Syrovatskii (1965) $\left(v \sim 20 N_{\mathrm{e}} / B\right.$, with $N_{\mathrm{e}} \sim$ $10^{6} \mathrm{~cm}^{-3}$ and $B=10^{-3}$ Gauss at the shock front), explaining the SED profile at $\sim 10^{11} \mathrm{~Hz}$. The unabsorbed bremsstrahlung explains the slope of the SED between about $10^{10}$ and $10^{11} \mathrm{~Hz}$. At $v<10^{10} \mathrm{~Hz}$ the bremsstrahlung shows the high slopes that are produced by free-free self-absorption. The optical depth $\tau=8.24 \times 10^{-2} T^{-1.35} v^{-2.1} E$ (where $T$ is to be measured in $\mathrm{K}$, $v$ in $\mathrm{GHz}$ and $E$, the so-called emission measure $E=\int n_{\mathrm{e}} n^{+} \mathrm{d} s$, in $\mathrm{cm}^{-6} \mathrm{pc}-$ Osterbrock 1988) is low for gas at relatively high temperatures; however, in the downstream nebulae of H1-36 we found large regions (i.e. large $\mathrm{d} s$ ) of gas at $T \sim 10^{4} \mathrm{~K}$ (Fig. 2), which make $\tau>1$. The other parameters that vary from slab to slab are the density, which is calculated through the compression equation, and the geometric thickness of the slab. At this point we would like to emphasise that the calculation of the density profile is not assumed a priori in our models accounting for shocks, but consistently calculated across the whole emitting nebula (Sect. 3.2). In this case, the thinner the slab, the better the approximation of the calculated spectra. Therefore the densities must be high enough to give reasonable absorption. The turnover corresponds to different frequencies for different slabs, and the final bremsstrahlung results from the integrated flux throughout the whole length of the nebula. The actual turnover frequency $v_{\mathrm{t}}$ indicates then the transition from the optically-thin to the optically-thick case.

It is worth highlighting that higher densities generally refer to the nebula downstream of the shock front facing the WD between the stars (model $m_{\mathrm{rev}}$ ), while the densities downstream of the expanding shock (model $m_{\text {exp }}$ ) correspond to an opticallythin nebula. Therefore, the radio spectrum observed in SS may be emitted by the gas between the stars, with a larger, more increasing contribution from the expanding nebula at lower frequencies. Interestingly, this agrees with the variable size of the radio nebula, which becomes gradually larger at lower frequencies (i.e. $0.6 \operatorname{arcsec}$ at $\lambda=2 \mathrm{~cm}$ and $5 \operatorname{arcsec}$ at $\lambda=6 \mathrm{~cm}-$ Taylor 1988).

\subsubsection{The IR continuum}

One of the most intriguing aspects of the symbiotic phenomenon in D types pertains to the dusty environment. As a matter of fact, these systems show a broad IR excess that has been attributed to emission from circumstellar dust since the first IR surveys. Early observations already showed that, in D type objects, the dust excesses have colour temperatures near $1000 \mathrm{~K}$ (Feast et al. 1983); nevertheless today, thanks to the IR astronomy developments, it has been realised that it is difficult to explain the observed IR spectrum by a single temperature. Theoretical models, too, confirmed that different "dust" temperatures should be combined to reproduce the NIR-MIR data (e.g. Anandarao et al. 1988; Schild et al. 2001; Angeloni et al. 2007c, in preparation).

In $\mathrm{H} 1-36$, the temperature of the dust shells is derived by modelling the IR data we collected, and quite surprisingly they agree in the overlapping frequency range. This proves that variability, even if present as in all symbiotic IR spectra, has not been high enough to substantially modify the dust continuum emission and then invalidate our results (see Sect. 2, Fig. 1, and Table 3).

It is worth noticing that the cooling rate is very strong at the densities typical of the region closer to the shock front downstream, which corresponds to the maximum temperature of dust. This implies that the flux from the shell corresponds mainly to the maximum temperature of the grains and can be modelled by a black body (bb). Figure 3 shows that the whole IR continuum can be explained by the combination of two bb curves, 
corresponding to temperatures of $800 \mathrm{~K}$ and $250 \mathrm{~K}$. By comparing the models with the data, we obtain the $\eta$ factors which depend on the radius of the dust shell $r$, and on the distance of the system to Earth $d$ ( $\left.\eta=r^{2} / d^{2}\right)$, being the fluxes calculated at the nebula and the data, obviously, measured on Earth. Adopting $d=4.5 \mathrm{kpc}$ (Allen 1983), we find $r=4.4 \times 10^{14} \mathrm{~cm}$ and $4.7 \times 10^{15} \mathrm{~cm}$ for the shells at $800 \mathrm{~K}$ and $250 \mathrm{~K}$, respectively. Both the derived inner shell temperature and radius are in good agreement with what was found by Allen (1983), who suggested that the cool component is heavily embedded in its own dust shell; specifically, the hot shell is circumstellar.

An interesting issue is related to the outer shells: if we were to rely on the Allen's proposed binary separation $(3 \times$ $\left.10^{16} \mathrm{~cm}\right)$, then even this shell would be circumstellar, namely, well-comprised within the system. Allen himself admitted such a large binary separation was striking, though; moreover, he stated that, translated into an angular separation of $\sim 0.5$ arcsec according to the suggested distance of $4.5 \mathrm{kpc}$, this binary separation would have been large enough to be resolved by careful infrared astrometry, as well as by high-resolution optical and radio observations. A few years later, Taylor (1988) succeeded in resolving the radio nebula. Conversely, Bhatt \& Sagar (1991) failed to detect any nebulosity in the optical emission lines, arguing that if this was actually present, it should be smaller than $\sim 2$ arcsec. Corradi et al. (1999) finally resolved the optical nebula and found out that it also appeared in a continuum image, therefore suggesting that it might be a reflection nebula.

At this point, we would like to stress that the unusually large size of the system has been constrained so far by observations of gaseous emitting nebulæ and that the binary stellar components have never been resolved. This might be a clue that the actual binary separation is overestimated, therefore implying that the outer shell we found directly by modelling the IR data may even be circumbinary, surrounding both the stars. This would not be so out of the ordinary for D-type SS, since this dichotomy in the dust shell distribution (with a hot shell being circumstellar, and a cool one circumbinary) has been confirmed by recent studies (Angeloni et al. 2007c, in preparation).

H1-36 appears as an extreme SS: only further observations at higher spatial resolution and in different spectral ranges will be able to investigate the actual size and physical properties of the dust and gas nebulæ.

\subsubsection{The UV continuum}

For the sake of completeness, we would like to note that longward of $\log v=14.5$ the UV data are well-fitted by a $T_{*}=150000 \mathrm{~K} \mathrm{bb}$ curve (Fig. 3), in agreement with Allen's (1983) derived hot star temperature. In that range, the WD flux dominates the bremsstrahlung emission. However, it is worth recalling that, between $\log v \sim 16.4$ and $\log v \sim 17.7$, absorption of X-rays is quite strong, implying that X-rays, if present, would be soft and mainly due to bremsstrahlung emission arising from the high-velocity component we deduce from the IR line features (see Sect. 4.3).

\section{3. $A$ jet in $H 1-36$ ?}

In a previous analysis of ISO SS infrared spectra (Angeloni et al. 2007a - hereafter Paper I), we found broad emission lines, indicating high-velocity components in many objects of the sample (Paper I - Fig. 1, Table 4). In H1-36, the FWHM of these line profiles corresponds to velocities of $500 \mathrm{~km} \mathrm{~s}^{-1}$. We recall that high-velocity components were not observed in the optical-UV spectra presented by Allen (1983), which showed $F W H M \leq 200 \mathrm{~km} \mathrm{~s}^{-1}$. Consequently, since the ISO-SWS spectrum shows several highly-ionized and broad $\mathrm{Ne}$ and $\mathrm{O}$ lines, incompatible with the physical models derived by the optical-UV spectra, we decided to include these new results in order to lead to as large and consistent interpretation as possible. We then calculated a further model, which is characterised by higher shock velocities and lower densities and able to reproduce the observed IR lines without making the previous fit worse.

In Paper I we found that the $[\mathrm{NeVI}] 7.65 \mu \mathrm{m}$ line dominates in H1-36, followed by the [OIV] $25.89 \mu \mathrm{m}$, [NeIII] $15.55 \mu \mathrm{m},[\mathrm{NeV}] 24.31 \mu \mathrm{m}$, and [NeV] $14.32 \mu \mathrm{m}$ lines. The model calculated with $V_{\mathrm{s}}=500 \mathrm{~km} \mathrm{~s}^{-1}, n_{0}=10^{4} \mathrm{~cm}^{-3}$, $U=8$, and $D=10^{16} \mathrm{~cm}$ reproduces the observed intensity ratio well: in fact, we obtain $[\mathrm{NeII}] 12.8 /[\mathrm{NeVI}] 7.65=0$, $[\mathrm{NeV}] 14.32 /[\mathrm{NeVI}] 7.65=0.47$, [NeIII] $15.55 /[\mathrm{NeVI}] 7.65=$ $1.3 \mathrm{e}-4$, and $[\mathrm{NeV}] 24.31 /[\mathrm{NeVI}] 7.65=0.05$, in agreement with the observed upper limits, respectively $[\mathrm{NeV}] 14.32 /[\mathrm{NeVI}] 7.65<0.5,[\mathrm{NeIII}] 15.55 /[\mathrm{NeVI}]<0.2$ and $[\mathrm{NeV}] 24.31 /[\mathrm{NeVI}]<0.15$. Moreover, the same model represents a non negligible contribution only on the high ionization level lines, particularly to CIV 1549, [NeV] 3425, [FeII]6087, and a relatively strong contribution to HeII 1640.

We can now calculate the distance of this high-velocity structure from the WD. A WD $T_{*}=150000 \mathrm{~K}$ corresponds to a ionizing photon flux of $4 \times 10^{26}$ photons $\mathrm{cm}^{-2} \mathrm{~s}^{-1}$. This flux is related to the ionization parameter and to the gas number density in the radiation dominated zone by $F_{v}\left(R_{\mathrm{WD}} / r_{j}\right)^{2}=U n c$. Adopting an average density of $n=10^{5} \mathrm{~cm}^{-3}$ after compression, and a WD radius of $10^{9} \mathrm{~cm}$, we obtain $\log \eta=-16.2$. This value allows us to place the bremsstrahlung emission from this component in the continuum SED diagram of Fig. 3 and to verify that its contribution to the radio spectrum is negligible when compared to the bremsstrahlung from the nebulæ. Conversely, on the high-energy side of the spectrum, it is interesting to notice that $V_{\mathrm{s}}=500 \mathrm{~km} \mathrm{~s}^{-1}$ would correspond to a temperature in the immediate postshock region of $\sim 3.6 \times 10^{6} \mathrm{~K}$, hence indicating that a soft X-ray emission may actually be present in $\mathrm{H} 1-36$.

Eventually, after this consistent analysis, we would like to stress the possibility that this high-velocity component in H1-36 is a jet-like feature. This suggestion, if confirmed, would be fascinating, as only recently it has been recognised that SS are a class of jet-producing objects. Furthermore, the two SS X-ray jets that have been discovered to date (R Aqr, Nichols et al. 2007 and $\mathrm{CH}$ Cyg, Karovska et al. 2007) both show an X-ray structure that is more extended than their radio ones (Sokoloski et al. 2006). This would explain why this $10^{16} \mathrm{~cm}$ jet has not been seen in the radio image (with comparable angular resolution) and drive us to indicate SS as promising X-ray targets.

\section{Concluding remarks}

In this paper we have analysed the spectra of D-type SS H1-36 within a colliding-wind theoretical framework. After having constrained the relative physical conditions by modelling more than 40 emission lines from radio to UV, we have been able to confidently explain the continuum SED by taking into account all the emitting contributions arising from both the stars, the dust shells, and the gaseous nebulæ. A comprehensive model of the radio spectra allowed reproduction of the different slopes of the radio profile and the turnover frequency, as well as the size of the observed nebulæ at several frequencies in light of the different contributions from the expanding and reverse shocks. The 
IR continuum unveiled the presence of two dust shells with characteristic radii and temperatures. The inner shell is confirmed to surround the Mira star, while the outer one may be circumbinary, i.e. surrounding the whole binary system. We believe that the presence of multiple dust shells is not a unique characteristic of H1-36, but it may somehow represent a distinctive feature of D-type SS. Furthermore, the broad profiles of the IR lines directed us to investigate whether a high-velocity component (perhaps an X-ray jet) may be present. This insight led us to indicate H1-36 as a promising X-ray target and represents a further support to the emerging interpretation of SS as a class of jet-producing objects. We then encourage new observations and studies which consistently take the complex nature of SS throughout the whole electromagnetic spectrum into account.

Acknowledgements. The authors are very grateful to the anonymous referee for many helpful comments that improved the readability of the paper. R.A. acknowledges the kind hospitality of the School of Physics \& Astronomy of Tel Aviv University.

\section{References}

Aaquist, O. B., \& Kwok, S. 1990, A\&AS, 84, 229

Aaquist, O. B., \& Kwok, S. 1991, ApJ, 378, 599

Allen, D. 1981, Nature, 293, 99

Allen, D. A. 1983, MNRAS, 204, 113

Allen, D. A., Hall, P. J., Norris, R. P., et al. 1989, MNRAS, 236, 363

Anandarao, B. G., Taylor, A. R., \& Pottasch, S. R. 1988, A\&A, 203, 361

Angeloni, R., Contini, M., Ciroi, S., \& Rafanelli, P. 2007a, AJ, 134, 205 (Paper I)

Angeloni, R., Contini, M., Ciroi, S., \& Rafanelli, P. 2007b, A\&A, accepted

Bastian, T. S. 1992, ApJ, 387, L77

Belczyński, K., Mikołajewska, J., Munari, R. J., Ivison, R. J., \& Friedjung, M. 2000, A\&AS, 146, 407

Bell, A. R. 1978a, MNRAS, 182, 147

Bell, A. R. 1978b, MNRAS, 182, 443

Bhatt, H. C., \& Sagar, R. 1991, J. A\&A, 12, 111

Bisikalo, D. V., Boyarchuk, A. A., Kilpio, E. Y., Tomov, N. A., \& Tomova, M. T. 2006, Astron. Rep., 50, 722

Contini, M. 1997, ApJ, 483, 887

Contini, M., \& Prialnik, D. 1997, ApJ, 475, 803

Contini, M., Collodel, L., \& Rafanelli, P. 1997, ASSL 218, Astronomical Time Series, 203

Contini, M., \& Formiggini, L. 1999, ApJ, 517, 925

Contini, M., \& Formiggini, L. 2001, A\&A, 375, 579

Contini, M., \& Formiggini, L. 2003, MNRAS, 339, 148

Contini, M. 2003, MNRAS, 339, 125

Contini, M. 2004, A\&A, 422, 591
Costa, R. D. D., \& de Freitas Pacheco, J. A. 1994, A\&A, 285, 998

Corradi, R. L. M., Brandi, E., Ferrer, O. E., \& Schwarz, H. E. 1999, A\&A, 343, 841

Cox, D. P. 1972, ApJ, 178, 143

Crocker, M. M., Davis, R. J., Eyres, S. P. S., et al. 2001, MNRAS, 326, 781

Egan, M. P., Price, S. D., \& Kraemer, K. E. 2003, BAAS, 35, 1301

Feast, M. W., Catchpole, R. M., Whitelock, P. A., Carter, B. S., \& Roberts, G. 1983, MNRAS, 203, 373

Formiggini, L., Contini, M., \& Leibowitz, E. M. 1995, MNRAS, 277, 1071

Giacconi, R., Murray, S., Gursky, H., et al. 1974, ApJS, 27, 37

Ginzburg, V. L., \& Syrovatskii, S. I. 1965, ARA\&A, 3, 297

Girard, T., \& Willson, L. A. 1987, A\&A, 183, 247

Haro, G. 1952, Boletin de los Observatorios Tonantzintla y Tacubaya, 1, 1

He, J. H., Szczerba, R., Chen, P. S., \& Sobolev, A. M. 2005, A\&A, 434, 201

Ivison, R. J., Seaquist, E. R., Schwarz, H. E., Hughes, D. H., \& Bode, M. F. 1995, MNRAS, 273, 517

Ivison, R. J., Seaquist, E. R., \& Hall, P. J. 1995, Ap\&SS, 224, 255

Ivison, R. J., Yates, J. A., \& Hall, P. J. 1998, MNRAS, 295, 813

Jones, P. A. 1985, MNRAS, 216, 613

Karovska, M., Carilli, C. L., Raymond, J. C., \& Mattei, J. A. 2007, [arXiv: astro-ph/0703278]

Kenny, H. T., \& Taylor, A. R. 2005, ApJ, 619, 527

Medina Tanco, G. A., \& Steiner, J. E. 1995, AJ, 109, 1770

Nichols, J. S., DePasquale, J., Kellogg, E., et al. 2007, [arXiv: astro-ph/0701336]

Nussbaumer, H., Schmutz, W., \& Vogel, M. 1995, A\&A, 293, L13

Nussbaumer, H. 2000, Thermal and Ionization Aspects of Flows from Hot Stars, ASP Conf. Ser., 204, 317

Osterbrock, D. E. 1988, in Astrophysics of Gaseous Nebulae and Active Galactic Nuclei (University Science Book)

Pereira, C. B. 1995, A\&AS, 111, 471

Pereira, C. B., Landaberry, S. J. C., \& Junqueira, S. 1998, A\&A, 333, 658

Purton, C. R., Allen, D. A., Feldman, P. A., \& Wright, A. E. 1977, MNRAS, $180,97 \mathrm{P}$

Purton, C. R., Feldman, P. A., Marsh, K. A., Allen, D. A., \& Wright, A. E. 1982, MNRAS, 198, 321

Ramos-Larios, G., \& Phillips, J. P. 2005, MNRAS, 357, 732

Schild, H., Eyres, S. P. S., Salama, A., \& Evans, A. 2001, A\&A, 378, 146

Seaquist, E. R., Taylor, A. R., \& Button, S. 1984, ApJ, 284, 202

Seaquist, E. R., Krogulec, M., \& Taylor, A. R. 1993, ApJ, 410, 260

Siodmiak, N., \& Tylenda, R. 2001, VizieR Online Data Catalog, 337, 31032

Sokoloski, J. 2006, Chandra Proposal, 2059

Taylor, A. R., \& Seaquist, E. R. 1984, ApJ, 286, 263

Taylor, A. R. 1988, ASSL, 145, IAU Colloq. 103, The Symbiotic Phenomenon, 77

Viegas, S. M., \& Contini, M. 1994, ApJ, 428, 113

Watson, S. K., Eyres, S. P. S., Davis, R. J., et al. 2000, MNRAS, 311, 449

Webster, B. L., \& Allen, D. A. 1975, MNRAS, 171, 171

Whitelock, P. A. 1987, PASP, 99, 573

Williams, R. E. 1967, ApJ, 147, 556

Wright, A. E., \& Barlow, M. J. 1975, MNRAS, 170, 41 